\title{
Primary sources of pelvic serous cancer in patients with endometrial intraepithelial carcinoma
}

\author{
Lin Jia ${ }^{1,2,6}$, Zeng Yuan ${ }^{1,2,6}$, Yiying Wang ${ }^{2,3}$, Janiel M Cragun ${ }^{4}$, Beihua Kong ${ }^{1}$ and \\ Wenxin Zheng $1,2,4,5$
}

${ }^{1}$ Department of Obstetrics and Gynecology, Qilu Hospital, Shandong University, Shandong, China;

${ }^{2}$ Department of Pathology, University of Arizona College of Medicine, Tucson, AZ, USA; ${ }^{3}$ Department of

Obstetrics and Gynecology, Henan Provincial People's Hospital, Henan, China; ${ }^{4}$ Department of Obstetrics and Gynecology, University of Arizona, Tucson, AZ, USA and ${ }^{5}$ Arizona Cancer Center, University of Arizona, Tucson, AZ, USA

\begin{abstract}
Serous endometrial intraepithelial carcinoma is often associated with extrauterine disease. It is currently unclear where does the extrauterine disease come from. This study addressed this issue. A total of 135 samples from 21 serous endometrial intraepithelial carcinoma patients were studied. Cellular lineage relationships between intrauterine and extrauterine serous carcinomas were determined by TP53-mutation analysis and correlated to the clinicopathologic features. There were three conditions contributing the extrauterine disease: metastasis from serous endometrial intraepithelial carcinoma $(n=10)$ showed identical TP53 mutation between intrauterine lesions and extrauterine disease, cases of adnexal origin $(n=5)$ had discordant TP53 mutations, and the mixed cellular origin cases $(n=6)$ with both identical and discordant mutation status. Patients with extrauterine disease from serous endometrial intraepithelial carcinoma metastasis typically had small tumor masses $(<\mathbf{2} \mathbf{c m})$ in extrauterine sites and without finding of serous tubal intraepithelial carcinoma, while extrauterine disease with adnexal or tubal origin commonly had larger tumor masses in extrauterine sites including ovary and omentum and serous tubal intraepithelial carcinoma. The majority of extrauterine diseases associated with serous endometrial intraepithelial carcinoma are metastasized from the endometrium. Serous endometrial intraepithelial carcinoma is frequently associated with serous cancers of adnexal or tubal origin, indicating that endometrial and adnexal or tubal serous cancers may share similar etiologies. TP53-mutation analysis provides a strong linkage for cellular lineage analysis. Tumor size in extrauterine disease and presence of serous tubal intraepithelial carcinoma or not are useful clinicopathologic features to determine primary cancer site, which helps in clinical management.
\end{abstract}

Modern Pathology (2015) 28, 118-127; doi:10.1038/modpathol.2014.76; published online 13 June 2014

Endometrial carcinoma, the most common malignancy of the female genital tract, was estimated at 52630 new cases and 8590 cancer-related deaths in the United States in $2014 .^{1}$ Endometrial carcinomas have been classified into two types. Type I, represented by endometrioid histology, arises commonly from endometrial hyperplasia or endometrial intraepithelial neoplasia and is mainly estrogen dependent. ${ }^{2,3}$ It accounts for $75-80 \%$ of endometrial

Correspondence: Dr W Zheng, MD, FCAP, Department of Pathology, University of Arizona College of Medicine, 1501 N. Campbell Avenue, \#5224A, Tucson, AZ 85724, USA.

E-mail: zhengw@email.arizona.edu

${ }^{6}$ These authors contributed equally to this work.

Received 28 January 2014; accepted 27 March 2014; published online 13 June 2014 cancers and is generally indolent with a favorable prognosis. In contrast, type II cancer is typically endometrial serous carcinoma, develops from resting or atrophic endometrium, and is rarely associated with estrogen stimulation. Although comprising only about $10 \sim 15 \%$ of endometrial cancers, endometrial serous carcinoma is known for its aggressive behavior and poor prognosis, accounting for more than $40 \%$ of endometrial cancer deaths. ${ }^{4,5}$

One reason for the poor prognosis of endometrial serous carcinoma is its association with extrauterine disease or pelvic serous carcinoma. Pelvic serous carcinoma is mainly derived from adnexa and endometrial serous carcinoma. ${ }^{6}$ Endometrial serous carcinoma without myometrial invasion, or serous endometrial intraepithelial carcinoma, is defined morphologically as the replacement of endometrial 
surface epithelium and/or glands by malignant serous cells. ${ }^{7-11}$ To some, serous endometrial intraepithelial carcinoma is synonymous with stage IA endometrial serous carcinoma or minimal uterine serous carcinoma when tumor size is less than $1 \mathrm{~cm} \cdot{ }^{12,13}$ In reported series of serous endometrial intraepithelial carcinoma, the incidence of extrauterine disease or pelvic serous carcinoma ranged from $33-67 \%$ (average approximately $50 \%$ )..$^{12-14}$ The exact origin of extrauterine serous carcinoma has yet to be established. Extrauterine disease was previously thought to be derived from transtubal metastasis. ${ }^{15}$ Alternative hypotheses include synchronous endometrial and adnexal origins as well as singular adnexal origin for both serous endometrial intraepithelial carcinoma and extrauterine disease. ${ }^{16-23}$ Identifying the cell of origin of extrauterine disease in patients with serous endometrial intraepithelial carcinoma may improve cancer detection, prevention, management, and optimally reduce the mortality of this fatal disease.

Genetic markers have commonly been used to trace the origin of a disease. TP53 alteration has been recognized as an early event in the process of endometrial serous carcinoma carcinogenesis. ${ }^{7,24}$ Our previous studies showed that endometrial serous carcinoma develops from endometrial glandular dysplasia and then to noninvasive serous endometrial intraepithelial carcinoma in a stepwise fashion through analyzing specific TP53 gene mutations. $^{7,25,26}$ TP53 point-mutation analysis has been applied to non-gynecologic cancers to define relationships between independent primaries versus metastasis. ${ }^{27,28}$ In this study, to determine the cell origin of extrauterine disease, we clarified the relationship between serous endometrial intraepithelial carcinoma and associated extrauterine disease by comparing and characterizing clinicopathologic features of representative cases and analyzing the mutational status of the TP53 gene in intrauterine and extrauterine sites.

\section{Materials and methods}

\section{Case Selection}

This study included 21 serous endometrial intraepithelial carcinoma patients with extrauterine disease who underwent surgical staging between 2006 and 2013 at the University of Arizona Medical Center, USA and Qilu Hospital, Shandong University, China. Tumor sizes and locations were described and recorded. Tissue specimens were histologically examined. The entire uterus was submitted to rule out invasive cancer for all serous endometrial intraepithelial carcinoma cases. Comprehensive evaluation of the fallopian tube was performed using SEE-FIM protocol. ${ }^{29,30}$ Extrauterine sites were sectioned with a 1 section/cm rule, except for the omentum. Omentum with no gross tumor was divided into 10 sections. Omentum with multiple tumor masses, individually measuring larger than $2 \mathrm{~cm}$ (omental cake), was divided into 2-3 sections. Diagnosis of serous endometrial intraepithelial carcinoma, endometrial serous carcinoma, and serous carcinomas involving fallopian tube and ovary was made based on criteria defined previously. ${ }^{10,31}$ Precancerous endometrial conditions were identified to include endometrial glandular dysplasia, tubal intraepithelial carcinoma in the fimbriated end and freefloating cancer cells within the tubal lumen. The study has been approved by Institutional Review Boards.

\section{Tissue Samples and Laser Capture Microdissection}

A total of 135 tumor samples from 21 serous endometrial intraepithelial carcinoma cases were retrieved. Thirty-six were from intrauterine sections containing serous endometrial intraepithelial carcinoma and 99 were from sections of extrauterine disease. All samples were derived from formalinfixed, paraffin-embedded tissue blocks. Serial 8- $\mu \mathrm{m}$ sections were mounted on PEN membrane slides (Arcturus Bioscience, Mountain View, CA, USA). A stained section of each specimen was reviewed to confirm histological presence of cancer. Targeted epithelia (500-1000 cells) were microdissected using the Veritas Laser Capture Microdissection System (Arcturus Bioscience) according to the standard protocols described previously. ${ }^{25}$

\section{TP53 Gene-Mutation Analysis}

All samples were screened for mutations in exon 5-8 of TP53 gene by touchdown PCR and subsequent DNA sequence analysis as described previously. ${ }^{25,26,32}$ Purified PCR products were sent to the Genomic Analysis and Technology Core Facility of the University of Arizona and both strands were sequenced using an Applied Biosystems 3730 DNA Analyzer. Candidate mutations were examined in the TP53 mutations database (http://p53.free.fr/ Database/p53_database.html).

Cellular lineage relationships between intrauterine and extrauterine serous carcinomas were determined by TP53 gene mutation analyses. Samples from a single patient demonstrating identical gene mutation(s) were considered to have a common clonal origin, whereas samples with different gene mutations were considered clonally independent. Cancers having both identical and discordant mutations among different endometrial and extrauterine disease samples were considered as mixed.

\section{TP53 Immunohistochemistry}

Immunohistochemistry for the presence of TP53 was performed as described previously. ${ }^{33,34}$ A total of 135 tumor samples were stained by TP53 mono- 
clonal antibody PAB1801 (Ab-2; Oncogene Science, Cambridge, MA, USA). Endometrial serous carcinoma from three patients with known TP53 alteration served as positive control. Negative controls were carried out by replacing primary antibodies with class-matched mouse IgG proteins on parallel sections. Sections were counterstained with hematoxylin. Immunostaining was repeated at least twice for each case. Qualitative assessment of immunohistochemical results for TP53 was based on distinct, strong nuclear staining as defined elsewhere. ${ }^{35}$ TP53 was considered positive when more than $75 \%$ of cancer nuclei stained and negative when $75 \%$ or less were stained. Occasional cytoplasmic and weak nuclear TP53 stainings were dismissed as insignificant. The staining result was given to each evaluated focus of targeted sample.

\section{Results}

\section{Clinicopathologic Features}

Age of patients ranged from 56-85 years (mean 68 years). Size of serous endometrial intraepithelial carcinoma or endometrial serous carcinoma without myometrial invasion measured from 0.2 (microscopic) to $3.0 \mathrm{~cm}$ (mean $0.8 \mathrm{~cm}$ ). Five cases involved an endometrial polyp, 12 cases were found in both endometrial polyp and non-polyp endometrium, and four cases were present in the superficial endometrium only. The location of extrauterine disease included fallopian tube (tubal mucosa, tubal fimbria, tubal serosa, and paratubal soft tissue), ovary (surface, superficial ovarian cortex, ovarian parenchyma, and hilum), omentum, peritoneum (pelvic side wall), pelvic and para-aortic lymph nodes, colon (colonic wall and serosa), and liver serosa. All but one patient (case 3) showed at least two extrauterine lesions involved either grossly or microscopically. Extrauterine tumor size ranged from 0.1 (microscopic) to larger than $10 \mathrm{~cm}$ (mean $5.6 \mathrm{~cm}$ ). When multiple tumor masses were present, the largest tumor was measured. There were 10 cases of extrauterine disease tumor size measuring $2 \mathrm{~cm}$ or smaller. All cancers found in extrauterine sites represented high-grade serous carcinoma except five cases showing focal areas of clear cell changes and one case with a focal area of sarcomatous change. Endometrial glandular dysplasia was found in $13(62 \%)$ of the 21 serous endometrial intraepithelial carcinoma cases. Serous tubal intraepithelial carcinoma was present in six cases, but no in situ carcinoma was found in either ovary or in the peritoneum.

\section{Cases of Extrauterine Disease with Identical TP53 Mutations as in Serous Endometrial Intraepithelial Carcinoma}

Multiple TP53 gene mutations were identified within exons 5-8. Among the 135 analyzed samples from 21 cases, 107 showed TP53 mutations in at least one exon. In the remaining 28 tumor samples, nine showed no mutation. The other 19 samples with exons noninformative for analysis were excluded from the study. Among the 21 cases, 10 (48\%) showed identical TP53 mutations between serous endometrial intraepithelial carcinoma and corresponding extrauterine disease. The extrauterine disease foci $(n=42)$ were obtained from ovary $(n=6)$, fallopian tube $(n=15)$, omentum $(n=11)$, peritoneum $(n=6)$, lymph node $(n=1)$, and colon serosa $(n=3)$. Identical mutations were found in exons $5(n=15), 6(n=2), 7(n=9)$, and $8(n=2)$. The detailed data are summarized in Table 1.

All 10 identical TP53 mutation cases were positive (diffuse and strong) for TP53 immunohistochemical staining. The correlation between serous endometrial intraepithelial carcinoma and extrauterine disease was $100 \%$ (Table 2).

Tumor size of extrauterine disease ranged from 0.3 to $4.5 \mathrm{~cm}$ (average $1.56 \mathrm{~cm}$ ). Tumor masses all measured smaller than $2 \mathrm{~cm}$ except one which was $4.5 \mathrm{~cm}$. Free-floating cancer cells in tubal lumen were found in three of the above cases. No serous tubal intraepithelial carcinoma or omental cake was identified. Representative images of serous endometrial intraepithelial carcinoma and corresponding free-floating cancer cells in tubal lumen are presented in Figure 1. The clinicopathologic features of the extrauterine disease are summarized in Table 3.

\section{Cases of Extrauterine Disease with Different TP53 Mutations as in Serous Endometrial Intraepithelial Carcinoma}

Among the 21 studied cases, there are five (24\%) which did not show identical TP53 mutations when foci of serous endometrial intraepithelial carcinoma $(n=9)$ and lesions of extrauterine disease $(n=24)$ were compared. The extrauterine diseases were derived from ovary $(n=4)$, fallopian tube $(n=8)$, omentum $(n=6)$, peritoneum $(n=4)$, colonic wall $(n=1)$, small-intestine serosa $(n=1)$. There are 16 effective point and five nonsense mutations. Among these five cases with different TP53 mutations, all five serous endometrial intraepithelial carcinoma and four extrauterine disease samples were positive for TP53 immunohistochemical staining. Only one extrauterine disease with five areas was negative, corresponding to a nonsense mutation (Table 2).

All five cases had multiple tumor masses in the extrauterine sites including omental cake. The tumor size from extrauterine sites ranged from 2.5 to $11.0 \mathrm{~cm}$ (average $7.83 \mathrm{~cm}$ ). Gross bilateral ovarian involvement was seen in four cases. Multiple pelvic and peritoneal sites were seen in all cases. Serous tubal intraepithelial carcinoma was found in two, while endometrial glandular dysplasia was seen in three cases (data not shown). The clinicopathologic features including tumor size, omental cake, 
Table 1 TP53 mutation analyses of SEIC with concurrent ExUD Cases of ExUD with identical TP53 mutations as in SEIC

\begin{tabular}{|c|c|c|c|}
\hline Case \# & $\begin{array}{l}\text { Tumor } \\
\text { location }\end{array}$ & Exon & $\begin{array}{l}\text { TP53 mutations of exons } \\
\text { 5-8 (codon_event) }\end{array}$ \\
\hline \multirow[t]{4}{*}{3} & E 1 & 7 & 248_CGG $>$ TGG $(\operatorname{Arg}>\operatorname{Trp})$ \\
\hline & E 2 & 7 & 248_CGG $>$ TGG (Arg $>$ Trp) \\
\hline & Om 1 & 7 & 248_CGG $>$ TGG (Arg $>$ Trp) \\
\hline & Om 2 & 7 & 248_CGG $>$ TGG (Arg $>$ Trp) \\
\hline \multirow[t]{7}{*}{5} & $\mathrm{E}$ & 6 & 193_CAT $>$ CTT (His > Leu) \\
\hline & TM & 6 & 193_CAT $>$ CTT $($ His $>$ Leu $)$ \\
\hline & $\mathrm{O}$ & & $\mathrm{NI} / \mathrm{N}$ \\
\hline & PT & 6 & 193_CAT $>$ CTT $($ His $>$ Leu) \\
\hline & Om 1 & & $\mathrm{~N}$ \\
\hline & Om 2 & & $\mathrm{~N}$ \\
\hline & LN & & $\mathrm{N}$ \\
\hline \multirow[t]{4}{*}{6} & $\mathrm{E}$ & 7 & 248_CGG $>$ TGG $($ Arg $>$ Trp) \\
\hline & $\mathrm{Om}$ & 7 & 248_CGG $>$ TGG (Arg $>$ Trp) \\
\hline & $\mathrm{P}$ & 7 & 248_CGG $>$ TGG (Arg $>$ Trp) \\
\hline & CS & 7 & 248_CGG $>$ TGG (Arg > Trp) \\
\hline \multirow[t]{7}{*}{7} & E 1 & 5 & 138_GCC $>$ CCC (Ala $>$ Pro) \\
\hline & E 2 & 5 & 138_GCC $>$ CCC (Ala $>$ Pro) \\
\hline & $\mathrm{O}$ & 5 & 138_GCC $>$ CCC (Ala $>$ Pro) \\
\hline & $\mathrm{TM}$ & 5 & 138_GCC $>$ CCC (Ala $>$ Pro) \\
\hline & TS & 5 & 138_GCC $>$ CCC (Ala $>$ Pro) \\
\hline & $\mathrm{Om}$ & 5 & 138_GCC $>$ CCC (Ala $>$ Pro) \\
\hline & $\mathrm{P}$ & & $\mathrm{NI} / \mathrm{N}$ \\
\hline \multirow[t]{4}{*}{8} & $\mathrm{E}$ & 7 & 257_CTG $>$ CAG $($ Leu $>$ Gln $)$ \\
\hline & $\mathrm{O}$ & 7 & 257_CTG > CAG $(\mathrm{Leu}>$ Gln $)$ \\
\hline & PT & 7 & 257_CTG $>$ CAG $(\mathrm{Leu}>$ Gln $)$ \\
\hline & $\mathrm{TL}$ & & $\mathrm{NI} / \mathrm{N}$ \\
\hline \multirow[t]{7}{*}{10} & E 1 & 5 & 143_GTG $>$ TTG $(\mathrm{Val}>\mathrm{Leu})$ \\
\hline & E 2 & 5 & 143_GTG $>$ TTG $(\mathrm{Val}>\mathrm{Leu})$ \\
\hline & TM & 5 & 143_GTG $>$ TTG $(\mathrm{Val}>\mathrm{Leu})$ \\
\hline & $\mathrm{O}$ & 5 & 143_GTG > TTG $(\mathrm{Val}>\mathrm{Leu})$ \\
\hline & PT & & $\mathrm{NI} / \mathrm{N}$ \\
\hline & $\mathrm{Om}$ & 5 & 143_GTG $>$ TTG $(\mathrm{Val}>\mathrm{Leu})$ \\
\hline & $\mathrm{P}$ & 5 & 143_GTG $>$ TTG $(\mathrm{Val}>\mathrm{Leu})$ \\
\hline \multirow[t]{8}{*}{12} & E 1 & 5 & 151_CCC $>$ CAC (Pro $>$ His) \\
\hline & E 2 & 5 & 151_CCC $>$ CAC (Pro $>$ His) \\
\hline & TM & 5 & 151_CCC $>$ CAC (Pro $>$ His) \\
\hline & $\mathrm{O}$ & 5 & 151_CCC $>$ CAC (Pro $>$ His $)$ \\
\hline & PT & 5 & 151_CCC $>$ CAC (Pro $>$ His) \\
\hline & $\mathrm{Om}$ & 5 & 151_CCC $>$ CAC (Pro $>$ His) \\
\hline & $\mathrm{P}$ & 5 & 151_CCC $>$ CAC (Pro $>$ His) \\
\hline & CS & & $\mathrm{NI} / \mathrm{N}$ \\
\hline \multirow[t]{6}{*}{13} & E 1 & 8 & 272_GTG $>$ CTG $(\mathrm{Val}>\mathrm{Leu})$ \\
\hline & E 2 & 8 & 272_GTG $>$ CTG $(\mathrm{Val}>\mathrm{Leu})$ \\
\hline & $\mathrm{TM}$ & & $\mathrm{NI} / \mathrm{N}$ \\
\hline & $\mathrm{Om}$ & 8 & 272_GTG $>$ CTG $(\mathrm{Val}>\mathrm{Leu})$ \\
\hline & $\mathrm{P}$ & 8 & 272_GTG $>$ CTG $(\mathrm{Val}>\mathrm{Leu})$ \\
\hline & CS & & $\mathrm{NI} / \mathrm{N}$ \\
\hline \multirow[t]{5}{*}{16} & E 1 & 5 & 175_CGC $>$ CAC (Arg $>$ His $)$ \\
\hline & E 2 & 5 & 175_CGC $>$ CAC (Arg $>$ His $)$ \\
\hline & TM & & $\mathrm{NI} / \mathrm{N}$ \\
\hline & PT & 5 & 175_CGC $>$ CAC \\
\hline & $\mathrm{Om}$ & 5 & 175_CGC $>$ CAC \\
\hline \multirow[t]{6}{*}{20} & $\mathrm{E}$ & 7 & 244_GGC $>$ GAC $($ Gly $>$ Asp $)$ \\
\hline & $\mathrm{O}$ & 7 & 244_GGC $>$ GAC (Gly $>$ Asp) \\
\hline & $\mathrm{TM}$ & & $\mathrm{NI} / \mathrm{N}$ \\
\hline & PT & 7 & 244_GGC $>$ GAC $($ Gly $>$ Asp) \\
\hline & $\mathrm{Om}$ & & $\mathrm{N}$ \\
\hline & $\mathrm{P}$ & & $\mathrm{N}$ \\
\hline
\end{tabular}

Cases of ExUD with different TP53 mutations as in SEIC

\begin{tabular}{|c|c|c|c|}
\hline Case \# & $\begin{array}{l}\text { Tumor } \\
\text { location }\end{array}$ & Exon & $\begin{array}{l}\text { TP53 mutations of exons } \\
\text { 5-8 (codon_event) }\end{array}$ \\
\hline \multirow[t]{5}{*}{9} & $\mathrm{E}$ & & $\mathrm{N}$ \\
\hline & $\mathrm{TM}$ & 8 & 277_TGT $>$ GGT (Cys $>$ Gly) \\
\hline & Om 1 & 8 & 277_TGT $>$ GGT (Cys $>$ Gly) \\
\hline & Om 2 & & $\mathrm{~N}$ \\
\hline & $\mathrm{P}$ & & $\mathrm{N}$ \\
\hline
\end{tabular}

Table 1 (Continued)

Cases of ExUD with different TP53 mutations as in SEIC

\begin{tabular}{|c|c|c|c|}
\hline Case \# & $\begin{array}{l}\text { Tumor } \\
\text { location }\end{array}$ & Exon & $\begin{array}{l}\text { TP53 mutations of exons } \\
5-8 \text { (codon_event) }\end{array}$ \\
\hline \multirow[t]{8}{*}{14} & E 1 & 7 & 248_CGG $>$ TGG $(\operatorname{Arg}>\operatorname{Trp})$ \\
\hline & E 2 & 7 & 248_CGG > TGG (Arg > Trp) \\
\hline & TM(STIC) & 6 & 198_GAA $>$ TAA $($ Glu $>$ Stop $)$ \\
\hline & $\mathrm{O}$ & 6 & 198_GAA $>$ TAA (Glu $>$ Stop) \\
\hline & PT & 6 & 198_GAA > TAA (Glu $>$ Stop) \\
\hline & $\mathrm{Om}$ & 6 & 198_GAA $>$ TAA (Glu $>$ Stop) \\
\hline & $\mathrm{P}$ & & $\mathrm{NI} / \mathrm{N}$ \\
\hline & CW & 6 & 198_GAA > TAA (Glu > Stop) \\
\hline \multirow[t]{7}{*}{15} & E 1 & 5 & 143_GTG > TTG (Val > Leu) \\
\hline & E 2 & 5 & 143_GTG $>$ TTG $(\mathrm{Val}>\mathrm{Leu})$ \\
\hline & $\mathrm{TF}$ & & $\mathrm{NI} / \mathrm{N}$ \\
\hline & $\mathrm{O}$ & 8 & 286_GAA > CGA $($ Glu $>$ Gly) \\
\hline & PT & & $\mathrm{NI} / \mathrm{N}$ \\
\hline & $\mathrm{Om}$ & 8 & 286_GAA > CGA $($ Glu > Gly) \\
\hline & $\mathrm{P}$ & & $\mathrm{NI} / \mathrm{N}$ \\
\hline \multirow[t]{8}{*}{17} & E 1 & 7 & 248_CGG > TGG (Arg > Trp) \\
\hline & E 2 & 7 & 248_CGG > TGG (Arg > Trp) \\
\hline & TM (STIC) & 5 & 155_ACC $>$ GCC $($ Thr $>$ Ala $)$ \\
\hline & $\mathrm{O}$ & 5 & 155_ACC $>$ GCC $($ Thr $>$ Ala $)$ \\
\hline & PT & & $\mathrm{NI} / \mathrm{N}$ \\
\hline & $\mathrm{Om}$ & 5 & 155_ACC $>$ GCC $(\mathrm{Thr}>$ Ala $)$ \\
\hline & $\mathrm{P}$ & & $\mathrm{NI} / \mathrm{N}$ \\
\hline & SS & 5 & 155_ACC $>$ GCC $(\mathrm{Thr}>$ Ala $)$ \\
\hline \multirow[t]{5}{*}{19} & E 1 & 7 & 248_CGG > TGG (Arg > Trp) \\
\hline & E 2 & & $\mathrm{~N}$ \\
\hline & TM & 8 & 277_TGT $>$ GGT (Cys $>$ Gly) \\
\hline & $\mathrm{O}$ & 8 & 277_TGT $>$ GGT (Cys $>$ Gly) \\
\hline & $\mathrm{Om}$ & 8 & 277_TGT $>$ GGT (Cys $>$ Gly) \\
\hline
\end{tabular}

Cases of ExUD with mixed TP53 mutations status as in SEIC

\begin{tabular}{|c|c|c|c|}
\hline Case \# & $\begin{array}{l}\text { Tumor } \\
\text { location }\end{array}$ & Exon & $\begin{array}{l}\text { TP53 mutations of exons } \\
5-8 \text { (codon_event) }\end{array}$ \\
\hline
\end{tabular}$$
1
$$

E 1
E 2

E 1

TM (STIC)

$\mathrm{O}$
$\mathrm{PT}$

PT
Om

$\mathrm{P}$

LN

E 2

2

E 2

TF (STIC)

PT

PT

$\mathrm{P}$

E 1

E 2

4

TF (STIC)

$\mathrm{O}$

Om

CS

E 1

E 2

$\mathrm{O}$ (left)

$\mathrm{O}$ (right)

TM (left)

PT (right)

$\mathrm{Om}$

LS

18

E 2

TM

$\mathrm{O}$
242_TGC $>$ TCC (Cys $>$ Phe)

242_TGC $>$ TCC (Cys $>$ Phe)

176_TGC $>$ TGA (Cys $>$ Stop)

242 TGC $>$ TCC (Cys $>$ Phe) $\mathrm{NI} / \mathrm{N}$

176_TGC $>$ TGA (Cys $>$ Stop) 242_TGC $>$ TCC (Cys $>$ Phe) 176_TGC $>$ TGA (Cys $>$ Stop) 248 CGG $>$ TGG (Arg $>$ Trp) 248_CGG $>$ TGG (Arg $>$ Trp) 281_GAC > GAT (Asp > Asp) 248_CGG $>$ TGG (Arg $>$ Trp) 281_GAC $>$ GAT $($ Asp $>$ Asp) 281 GAC $>$ GAT $($ Asp $>$ Asp $)$ 281_GAC $>$ GAT $($ Asp $>$ Asp) 248_CGG > TGG (Arg > Trp) 248_CGG $>$ TGG $($ Arg $>$ Trp $)$ $220 \_$TAT $>$TGT $($Tyr $>$Cys $)$ 220 TAT $>$ TGT $($ Tyr $>$ Cys $)$ 248_CGG > TGG (Arg > Trp) $220 \_$TAT $>$TGT $($Tyr $>$Cys $)$ 220_TAT $>$ TGT $($ Tyr $>$ Cys $)$ 138_GCC $>$ CCC (Ala $>$ Pro) 138_GCC $>$ CCC (Ala $>$ Pro) 238_TGT $>$ TGA (Cys $>$ Stop) 138_GCC $>$ CCC (Ala $>$ Pro) 238_TGT $>$ TGA (Cys $>$ Stop) 138_GCC > CCC (Ala > Pro) $\mathrm{NI} / \mathrm{N}$

238_TGT $>$ TGA (Cys $>$ Stop) 205_TAT $>$ TGT $($ Tyr $>$ Cys $)$ 205_TAT $>$ TGT $($ Tyr $>$ Cys $)$ 275_TGT $>$ TTT (Cys $>$ Phe) 205_TAT $>$ TGT $($ Tyr $>$ Cys $)$ 238_TGT $>$ TGA (Cys $>$ Stop) $\mathrm{NI} / \mathrm{N}$ 
Table 1 (Continued)

Cases of ExUD with mixed TP53 mutations status as in SEIC

\begin{tabular}{cccc}
\hline Case \# & $\begin{array}{c}\text { Tumor } \\
\text { location }\end{array}$ & Exon & $\begin{array}{c}\text { TP53 mutations of exons } \\
\text { 5-8 (codon_event) }\end{array}$ \\
\hline \multirow{4}{*}{21} & Om & 8 & 275_TGT $>$ TTT (Cys $>$ Phe) \\
& P & 8 & 275_TGT $>$ TTT (Cys $>$ Phe) \\
E & 7 & 248_CGG $>$ TGG (Arg $>$ Trp) \\
& TM & 5 & 143_GTG $>$ TTG (Val $>$ Leu) \\
O & 5 & 143_GTG $>$ TTG (Val $>$ Leu) \\
& PT & 7 & 248_CGG $>$ TGG (Arg $>$ Trp) \\
& Om & 5 & 143_GTG $>$ TTG (Val $>$ Leu) \\
P & & NI/N
\end{tabular}

Abbreviations: SEIC, serous endometrial intraepithelial carcinoma; ExUD, extrauterine disease; STIC, serous tubal intraepithelial carcinoma; E, endometrium; TM, tubal mucosa; TF, tubal fimbria; TS, tubal serosa; PT, paratubal soft tissue; O, ovary; Om, omentum; P, peritoneum; LN, lymph node; CS, colon serosa; CW, colonic wall; SS, smallintestine serosa; LS, liver serosa; $\mathrm{N}$, negative; NI/N, noninformative/ negative.

$\mathrm{N}$ : all the exons did not show any TP53 mutations. NI/N: in this tumor sample, some of the exons were noninformative for analysis, while the others screened were negative for TP53 mutations.

Table 2 The relationship between TP53 mutation and its protein overexpression

\begin{tabular}{|c|c|c|c|c|c|}
\hline & \multirow{2}{*}{$\begin{array}{l}\text { \#Cases } \\
\text { (\#Foci) }\end{array}$} & \multicolumn{2}{|c|}{ \#TP53 mutations } & \multicolumn{2}{|c|}{ \#TP53 IHC+ } \\
\hline & & $P M(\%)$ & $N M(\%)$ & $P M(\%)$ & $N M(\%)$ \\
\hline IM & $10(58)$ & $44(76 \%)$ & $0(0)$ & $44(76 \%)$ & $0(0)$ \\
\hline DM & $5(33)$ & $18(55 \%)$ & $5(15 \%)$ & $18(64 \%)$ & $0(0)$ \\
\hline MM & $6(44)$ & $33(75 \%)$ & $7(16 \%)$ & $33(89 \%)$ & $0(0)^{\mathrm{a}}$ \\
\hline
\end{tabular}

Abbreviations: PM, point mutation; NM, nonsense mutation.

IM, cases of extrauterine disease with identical TP53 mutations as in serous endometrial intraepithelial carcinoma; DM, cases of extrauterine disease with different TP53 mutations from serous endometrial intraepithelial carcinoma; MM, cases of extrauterine disease with both identical and different TP53 mutations compared by serous endometrial intraepithelial carcinoma; \#TP53 mutations: representing the total number of mutations detected from exons 5 to 8; \#TP53 IHC + : representing the total number of positively immunohistochemistry-stained cancer foci studied. The percentage is computed by the total number of foci studied.

${ }^{\mathrm{a}}$ One focus from extrauterine disease stained nuclei strongly in about $40 \%$ of the cancer cells and an additional $30 \%$ weak stainings. As it stained $<75 \%$ of the nuclei, we considered it as negative.

free-floating cancer cells in tubal lumen, and presence of serous tubal intraepithelial carcinoma are summarized in Table 3.

\section{Cases of Extrauterine Disease with Mixed TP53 Mutations Status as in Serous Endometrial Intraepithelial Carcinoma}

In addition to cases with metastasis from serous endometrial intraepithelial carcinoma and independent primaries, we also revealed the presence of both identical and discordant TP53 gene mutations between intrauterine and extrauterine disease samples in six $(29 \%)$ of the 21 cases. Forty-four cancer foci were analyzed. Eleven were from serous endometrial intraepithelial carcinoma and 33 were from extrauterine disease. The mutations found in foci of serous endometrial intraepithelial carcinoma within the same uterus were identical. The 33 foci of extrauterine disease were derived from the ovary $(n=7)$, fallopian tube $(n=12)$, omentum $(n=6)$, peritoneum $(n=5)$, lymph node $(n=1)$, liver serosa $(n=1)$, and colon serosa $(n=1)$. Among these, there were 29 TP53 mutations, 22 point and 7 nonsense mutations. One group of mutations was identical to those found in corresponding serous endometrial intraepithelial carcinoma, whereas the other group was different from that in serous endometrial intraepithelial carcinoma but identical for some of those foci of extrauterine disease within the same patient.

In case 1, a point mutation was present in both serous endometrial intraepithelial carcinoma and in samples of the ovary and peritoneum. However, another point mutation was found in fallopian tube, omentum, and lymph node. This particular point mutation was not seen in serous endometrial intraepithelial carcinoma. The tubal sample was obtained from tubal serous intraepithelial carcinoma. A similar finding was found in two other cases. In case 11, a point mutation was identified in samples of serous endometrial intraepithelial carcinoma and the right ovary as well as right paratubal soft tissue. However, another point mutation was found among the samples of the left ovary, left tubal soft tissue, omentum, and liver serosa (Table 1).

Among the 44 cancer foci from the six cases, we identified $33(75 \%)$ point and $7(16 \%)$ nonsense mutations. The point-mutation foci generated strong diffuse positive TP53 staining, while nonsense mutation foci were correlated with negative immunohistochemical staining (Table 2).

The extrauterine tumor size of these cases ranges from 0.3 to $10 \mathrm{~cm}$ (average $3.37 \mathrm{~cm}$ ). Omental cake was seen in two of the six cases. Three cases had serous tubal intraepithelial carcinoma, while two cases showed free-floating cancer cells in tubal lumen (Table 3). Interestingly, the area of serous tubal intraepithelial carcinoma showed TP53 mutations different from those in corresponding serous endometrial intraepithelial carcinoma, but identical to some of those foci from corresponding extrauterine disease. Furthermore, the cases with serous tubal intraepithelial carcinoma all had omental cake and ovarian mass larger than $2 \mathrm{~cm}$ in size.

\section{Discussion}

Pelvic serous carcinoma is a general term of any high-grade serous carcinoma in the pelvis, mainly including serous cancers derived from ovary, peritoneum, fallopian tube, endometrium, and cervix. ${ }^{36-38}$ Pelvic serous carcinoma typically presents as 
a
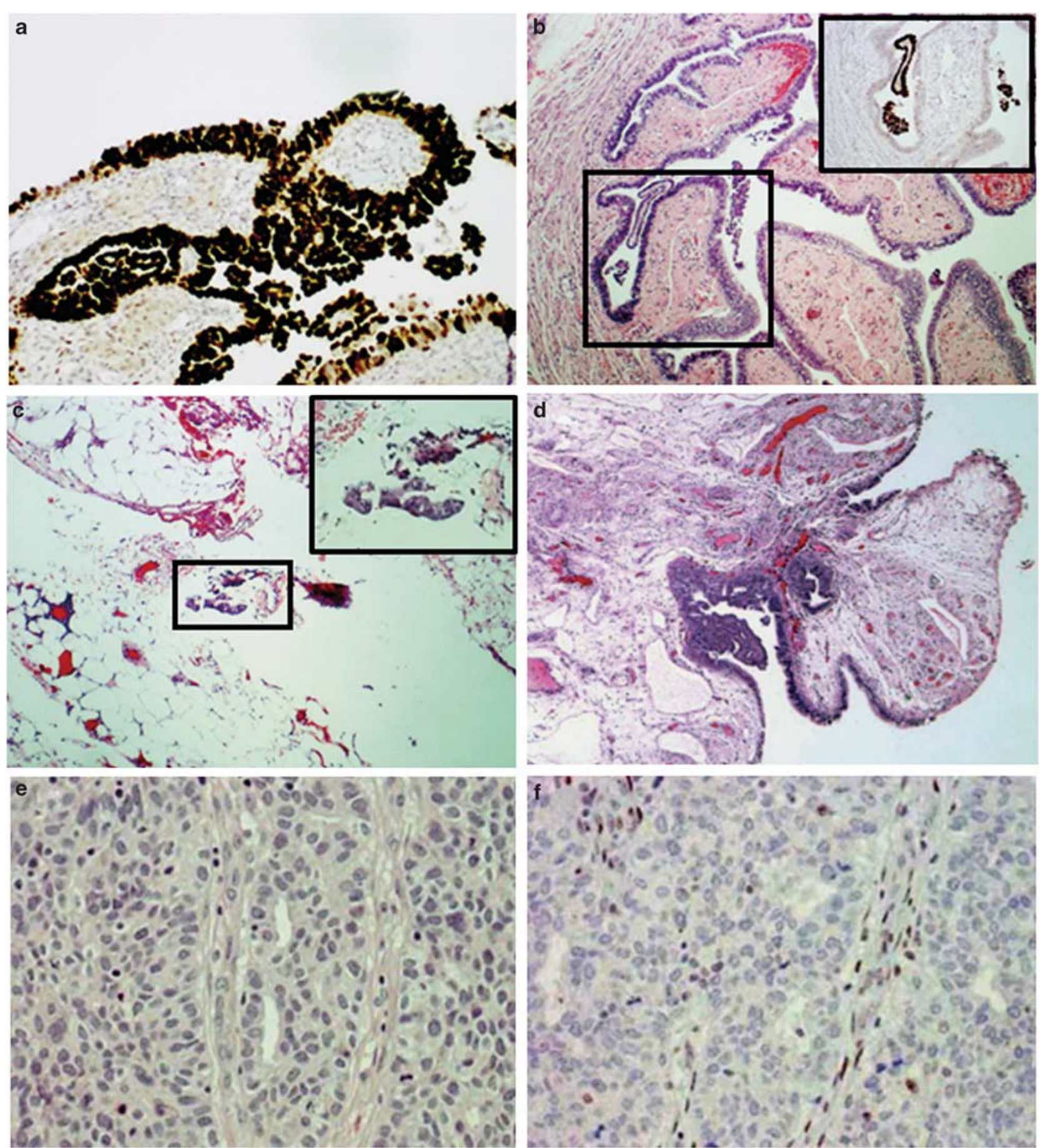

Figure 1 (a) The TP53 immunohistochemcal staining of serous endometrial intraepithelial carcinoma from case $3(\times 100)$. (b) A section of the fallopian tube from case 7 shows free-floating cancer cells in tubal lumen (H\&E, $\times 40)$. The cancer glandular cells within the tubal lumen (squared area) are clearly demonstrated by TP53 staining (inserted in upper right corner, $\times 40$ ). (c) Microscopic metastasis of serous carcinoma is found in the omentum $(\mathrm{H} \& \mathrm{E}, \times 40)$ from the same case 7 . This microscopic metastasis is magnified in the upper right corner $(\times 100)$. (d) An example of serous tubal intraepithelial carcinoma (H\&E, $\times 40)$ from case 1 with TP53 nonsense mutation of exon 5 at codon 176 from TGC to TGA (Cys to Stop). (e) The corresponding section from a 5-cm omental lesion of case 1 demonstrates high-grade serous carcinoma (H\&E, $\times 100)$. (f) The carcinoma in panel e shows negative TP53 staining. A weak staining was observed in stromal cells $(\times 100)$.

carcinomatosis with multiple foci of serous cancer involving the ovary, tubal and paratubal soft tissue, omentum, peritoneal surface, and serosal surface of many intrapelvic and abdominal organs. The cell origin of pelvic serous carcinoma has been the subject of research for several decades. Current 
Table 3 The relationship among tumor size, presence of STIC, and free-floating cancer cells in tubal lumen

\begin{tabular}{lccccc}
\hline & & $\begin{array}{c}\text { Tumor size, } \mathrm{cm} \\
\text { (average } \pm \text { s.d.) }\end{array}$ & $\begin{array}{c}\text { Omental } \\
\text { cake }\end{array}$ & \#STIC & \#FFCCTL \\
\hline $\mathrm{IM}$ & 10 & $1.56 \pm 0.41$ & $0 \%$ & $0 \%$ & $30 \%$ \\
$\mathrm{DM}$ & 5 & $7.83 \pm 3.24$ & $100 \%$ & $40 \%$ & $0 \%$ \\
$\mathrm{MM}$ & 6 & $3.37 \pm 1.82$ & $33 \%$ & $50 \%$ & $33 \%$ \\
\hline
\end{tabular}

Abbreviations: STIC, serous tubal intraepithelial carcinoma; FFCCTL, free-floating cancer cells in tubal lumen.

IM, cases of extrauterine disease with identical TP53 mutations as in serous endometrial intraepithelial carcinoma; DM, cases of extrauterine disease with different TP53 mutations from serous endometrial intraepithelial carcinoma; MM, cases of extrauterine disease with both identical and different TP53 mutations compared by serous endometrial intraepithelial carcinoma.

understanding is that pelvic serous carcinoma is mainly derived from fallopian tube and some from endometrial serous carcinoma. ${ }^{18,31,39}$ Origination from the ovary or the peritoneum cannot be currently defined, as no precancerous lesions have been found within these organ sites. ${ }^{32,40}$ Origination from the endocervix is negligible due to its extremely low incidence of endocervical serous carcinoma. ${ }^{41-43}$ Pelvic serous carcinoma, in the presence of endometrial serous carcinoma, particularly serous endometrial intraepithelial carcinoma, is equivalent to serous endometrial intraepithelial carcinoma associated with extrauterine disease. ${ }^{16}$ Identifying the cellular source for extrauterine disease associated with serous endometrial intraepithelial carcinoma as well as the cell of origin may benefit patient care.

In the current study, we found that the cellular source of the serous endometrial intraepithelial carcinoma-associated extrauterine disease is more complicated than previously thought. Through TP53 gene-mutation analysis, there appear to be three categories of origin: (1) metastasis from serous endometrial intraepithelial carcinoma, (2) adnexal origin, and (3) mixed origin (both serous endometrial intraepithelial carcinoma and adnexal). Mutations of TP53 have been detected in a diverse array of tumor types. TP53 is the most commonly altered tumor suppressor gene in human malignancies to date. ${ }^{16,44}$ Approximately $90 \%$ of TP53 mutations occur in exons 5-8. ${ }^{44}$ TP53 alteration has a key role in endometrial serous carcinoma carcinogenesis. ${ }^{6,38,45}$ Studies have shown high prevalence of TP53 mutations in endometrial serous carcinoma and its noninvasive form serous endometrial intraepithelial carcinoma (up to $90 \%$ and $80 \%$, respectively). ${ }^{24,26,36}$ TP53-mutation analysis well defines the cellular lineage between primary organ sites and potential metastasis including serous endometrial intraepithelial carcinoma and associated extrauterine disease. ${ }^{16,20,22,27,46}$ In 10 of the 21 serous endometrial intraepithelial carcinoma cases, multiple TP53 gene mutations were identified and identical mutations were found between the foci of serous endometrial intraepithelial carcinoma and their associated extrauterine disease, supporting that the extrauterine disease represents metastasis from corresponding serous endometrial intraepithelial carcinomas. In contrast, 5 of the 21 serous endometrial intraepithelial carcinoma cases showed discordant TP53 mutations between intrauterine and extrauterine samples, suggesting that associated extrauterine disease in those samples was not derived from the metastatic serous endometrial intraepithelial carcinoma but rather from the tubal origin. Six of the 21 cases showed some identical and some different TP53 gene mutations between intrauterine and extrauterine disease, indicating associated extrauterine disease had both endometrial and tubal origins. Immunohistochemically, the majority of samples from serous endometrial intraepithelial carcinoma and extrauterine disease containing TP53 point mutations showed strong diffuse nuclei staining, while nonsense mutations were completely negative. This is consistent with the well-recognized TP53 staining 'all or none' phenomenon. ${ }^{36}$

In addition to the TP53 alterations among the foci of serous endometrial intraepithelial carcinoma and their corresponding extrauterine disease, we analyzed clinicopathologic features of each case identifying clinically relevant associations. Gross tumor and tumor size found at the extrauterine sites were associated with the cell origin of extrauterine disease. Pelvic serous carcinoma typically presents as carcinomatosis. We measured the largest discrete tumors for each case, and correlated this and other clinicopathologic features to the TP53 mutation status. Serous endometrial intraepithelial carcinoma patients with extrauterine disease from a metastatic origin have tumors typically smaller than $2 \mathrm{~cm}$. Thirty percent of such cases had identifiable freefloating cancer cells in tubal lumen, but were without omental cake or serous tubal intraepithelial carcinoma. The findings suggest that serous endometrial intraepithelial carcinoma cases are able to metastasize to the pelvis or the abdomen through the fallopian tube. Transtubal cancer spread has been demonstrated previously. ${ }^{16,20,21,39}$ This may explain the high incidence of extrauterine disease in serous endometrial intraepithelial carcinoma cases, although the detailed molecular mechanisms remain to be clarified.

Extrauterine disease associated with serous endometrial intraepithelial carcinoma can be derived from coexistent tubal or adnexal high-grade serous cancer. The extrauterine disease of such tubal origin have different clinicopathologic features from serous endometrial intraepithelial carcinoma metastasis. Specifically, features include extrauterine tumor masses larger than $2 \mathrm{~cm}$, frequent omental cake formation, presence of serous tubal intraepithelial carcinoma, and rare free-floating cancer cells in tubal lumen. The finding of coexisting serous 
endometrial intraepithelial carcinoma and highgrade serous carcinoma of tubal origin suggests that endometrial and tubal serous cancers may share similar risk factors. It is accepted that the presence of serous tubal intraepithelial carcinoma in the tubal fimbria is a strong evidence of tubal origination for those high-grade serous cancers found within the pelvis. ${ }^{47-49}$ This is supported by the identical TP53 mutations identified between the foci of serous tubal intraepithelial carcinoma and the extrauterine disease in this study. A few cases of coexisting serous endometrial intraepithelial carcinoma and serous tubal intraepithelial carcinoma have been reported..$^{18}$ Jarboe et $a l^{18}$ has proposed a possibility of serous endometrial intraepithelial carcinoma origination from those serous tubal intraepithelial carcinoma cells through an assumed 'drop metastasis' process. The concept of drop metastasis is typically used in the clinical setting when isolated intramucosal cancer is seen in the endocervical mucosa, while the bulky cancer is present in the endometrium. It may also apply to a small amount of intraendometrial cancer found in association with large amount of cancer in the ovary or the fallopian tube. However, in these special situations, there must be no precancerous lesions found adjacent to the intramucosal tumors. In this study, the mechanism of drop metastasis appears unlikely, because the presence of endometrial glandular dysplasia, the precancerous lesion of serous endometrial intraepithelial carcinoma or endometrial serous carcinoma, was present in $62 \%$ of the serous endometrial intraepithelial carcinoma cases. Presence of endometrial glandular dysplasia adjacent to the area of serous endometrial intraepithelial carcinoma strongly argues against 'drop metastasis' in this setting. Meanwhile, different TP53 gene mutations found between serous endometrial intraepithelial carcinoma and serous tubal intraepithelial carcinoma support that they represent independent processes from different locations within the müllerian tract rather than a detachment and implantation of each other. However, metastasis, not necessary as an intramucosal lesion, by cellular detachment does happen, as we have noted that free-floating cancer cells in tubal lumen is more commonly seen in extrauterine disease derived from serous endometrial intraepithelial carcinoma than that of tubal origin, although the mechanism of the cellular detachment from these serous cancers remains to be clarified.

The cellular source of extrauterine disease can originate from associated serous endometrial intraepithelial carcinoma as well as high-grade serous cancers of tubal origin simultaneously. This was supported by the six mixed cases identified in this study. The clinicopathologic features could be the combined findings of the two described conditions. Careful evaluation of the entire endometrial cavity ${ }^{21}$ as well as the fallopian tube by using SEE-FIM protocol will help determine the cancer cellular lineage through identification of precancerous lesions and early cancers.

In summary, the cellular sources of serous endometrial intraepithelial carcinoma-associated extrauterine disease include metastasis from serous endometrial intraepithelial carcinoma through fallopian tube, high-grade serous carcinoma of tubal or adnexal origin, and a mixture of the two conditions. Future studies on the relationship between serous endometrial intraepithelial carcinoma and serous tubal intraepithelial carcinoma as well as their corresponding cancers and associated risk factors are needed. Further, studies focused on early detection and risk reduction will be more beneficial for patient care.

\section{Acknowledgments}

The project was supported in part by Better Than Ever Fund, Arizona Cancer Center Supporting Grant, P30 CA23074 from Arizona Cancer Center and Department of Pathology, University of Arizona Startup fund to WZ, by National Natural Science Foundation of China (no. 30901590) to LJ and by National Natural Science Foundation of China (no.81272857), Taishan Scholars Foundation of Shandong Province (No.ts20070743) to BK.

\section{Disclosure/conflict of interest}

The authors declare no conflict of interest.

\section{References}

1 Siegel R, Ma J, Zou Z, et al. Cancer statistics, 2014. CA: Cancer J Clin 2014;64:9-29.

2 Jarboe EA, Mutter GL. Endometrial intraepithelial neoplasia. Semin Diagn Pathol 2010;27:215-225.

3 Mutter GL. Endometrial intraepithelial neoplasia (EIN): will it bring order to chaos? The Endometrial Collaborative Group. Gynecol Oncol 2000;76:287-290.

4 Fader AN, Drake RD, O’Malley DM, et al. Platinum/ taxane-based chemotherapy with or without radiation therapy favorably impacts survival outcomes in stage I uterine papillary serous carcinoma. Cancer 2009;115: 2119-2127.

5 Havrilesky LJ, Secord AA, Bae-Jump V, et al. Outcomes in surgical stage I uterine papillary serous carcinoma. Gynecol Oncol 2007;105:677-682.

6 Zheng WX, Fadare O. Fallopian tube as main source for ovarian and pelvic (non-endometrial) serous carcinomas. Int J Clin Exp Patho 2012;5:182-186.

7 Zheng W, Xiang L, Fadare O, et al. A proposed model for endometrial serous carcinogenesis. Am J Surg Pathol 2011;35:e1-e14.

8 Fadare $\mathrm{O}$, Zheng WX. Insights into endometrial serous carcinogenesis and progression. Int J Clin Exp Patho 2009;2:411-432.

9 Spiegel GW. Endometrial carcinoma in situ in postmenopausal women. Am J Surg Pathol 1995;19: 417-432. 
10 Ambros RA, Sherman ME, Zahn CM, et al. Endometrial intraepithelial carcinoma: a distinctive lesion specifically associated with tumors displaying serous differentiation. Hum Pathol 1995;26:1260-1267.

11 Fadare O, Zheng W. Endometrial serous carcinoma (uterine papillary serous carcinoma): precancerous lesions and the theoretical promise of a preventive approach. Am J Cancer Res 2012;2:335-339.

12 Zheng WX, Schwartz PE. Serous EIC as an early form of uterine papillary serous carcinoma: recent progress in understanding its pathogenesis and current opinions regarding pathologic and clinical management. Gynecol Oncol 2005;96:579-582.

13 Wheeler DT, Bell KA, Kurman RJ, et al. Minimal uterine serous carcinoma-diagnosis and clinicopathologic correlation. Am J Surg Pathol 2000;24:797-806.

14 Slomovitz BM, Burke TW, Eifel PJ, et al. Uterine papillary serous carcinoma (UPSC): a single institution review of 129 cases. Gynecol Oncol 2003;91:463-469.

15 Snyder MJ, Bentley R, Robboy SJ. Transtubal spread of serous adenocarcinoma of the endometrium: an underrecognized mechanism of metastasis. Int J Gynecol Pathol 2006;25:155-160.

16 Roelofsen T, van Kempen LCLT, van der Laak JAWM, et al. Concurrent endometrial intraepithelial carcinoma (EIC) and serous ovarian cancer can EIC be seen as the precursor lesion? Int J Gynecol Cancer 2012;22: 457-464.

17 Yan Z, Hui P. Minimal uterine serous carcinoma with extrauterine tumor of identical morphology: an immunohistochemical study of 13 cases. Appl Immunohistochem Mol Morphol 2010;18:75-79.

18 Jarboe EA, Miron A, Carlson JW, et al. Coexisting intraepithelial serous carcinomas of the endometrium and fallopian tube: frequency and potential significance. Int J Gynecol Pathol 2009;28:308-315.

19 Hui P, Kelly M, O’Malley DM, et al. Minimal uterine serous carcinoma: a clinicopathological study of 40 cases. Mod Pathol 2005;18:75-82.

20 Baergen RN, Warren CD, Isacson C, et al. Early uterine serous carcinoma: clonal origin of extrauterine disease. Int J Gynecol Pathol 2001;20:214-219.

21 Soslow RA, Pirog E, Isacson C. Endometrial intraepithelial carcinoma with associated peritoneal carcinomatosis. Am J Surg Pathol 2000;24:726-732.

22 Kupryjanczyk J, Thor AD, Beauchamp R, et al. Ovarian, peritoneal, and endometrial serous carcinoma: clonal origin of multifocal disease. Mod Pathol 1996;9:166-173.

23 Goff BA, Kato D, Schmidt RA, et al. Uterine papillary serous carcinoma: patterns of metastatic spread. Gynecol Oncol 1994;54:264-268.

24 Tashiro $\mathrm{H}$, Isacson $\mathrm{C}$, Levine $\mathrm{R}$, et al. p53 gene mutations are common in uterine serous carcinoma and occur early in their pathogenesis. Am J Pathol 1997;150:177-185.

25 Zhang X, Liang SX, Jia L, et al. Molecular identification of 'latent precancers' for endometrial serous carcinoma in benign-appearing endometrium. Am J Pathol 2009;174:2000-2006.

26 Jia L, Liu Y, Yi X, et al. Endometrial glandular dysplasia with frequent p53 gene mutation: a genetic evidence supporting its precancer nature for endometrial serous carcinoma. Clin Cancer Res 2008;14:2263-2269.

27 Hodges KB, Cummings OW, Saxena R, et al. Clonal origin of multifocal hepatocellular carcinoma. Cancer 2010;116:4078-4085.
28 Jones TD, Eble JN, Wang M, et al. Clonal divergence and genetic heterogeneity in clear cell renal cell carcinomas with sarcomatoid transformation. Cancer 2005;104:1195-1203.

$29 \mathrm{Li}$ J, Fadare O, Xiang L, et al. Ovarian serous carcinoma: recent concepts on its origin and carcinogenesis. J Hematol Oncol 2012;5:8.

30 Medeiros F, Muto MG, Lee Y, et al. The tubal fimbria is a preferred site for early adenocarcinoma in women with familial ovarian cancer syndrome. Am J Surg Pathol 2006;30:230-236.

31 Przybycin CG, Kurman RJ, Ronnett BM, et al. Are all pelvic (nonuterine) serous carcinomas of tubal origin? Am J Surg Pathol 2010;34:1407-1416.

32 Kindelberger DW, Lee Y, Miron A, et al. Intraepithelial carcinoma of the fimbria and pelvic serous carcinoma: evidence for a causal relationship. Am J Surg Pathol 2007;31:161-169.

33 Zheng WX, Sung CJ, Cao PQ, et al. Early occurrence and prognostic significance of p53 alteration in primary carcinoma of the fallopian tube. Gynecol Oncol 1997;64:38-48.

34 Zheng WX, Cao PQ, Zheng M, et al. p53 overexpression and bcl-2 persistence in endometrial carcinoma: comparison of papillary serous and endometrioid subtypes. Gynecol Oncol 1996;61:167-174.

35 Zheng WX, Khurana R, Farahmand S, et al. p53 immunostaining as a significant adjunct diagnostic method for uterine surface carcinoma-precursor of uterine papillary serous carcinoma. Am J Surg Pathol 1998;22:1463-1473.

36 Kobel M, Reuss A, du Bois A, et al. The biological and clinical value of p53 expression in pelvic high-grade serous carcinomas. J Pathol 2010;222:191-198.

37 Salvador S, Gilks B, Kobel M, et al. The fallopian tube: primary site of most pelvic high-grade serous carcinomas. Int J Gynecol Cancer 2009;19:58-64.

38 Crum CP. Intercepting pelvic cancer in the distal fallopian tube: theories and realities. Mol Oncol 2009;3:165-170.

39 Massuger L, Roelofsen T, van Ham M, et al. The origin of serous ovarian cancer may be found in the uterus: a novel hypothesis. Med Hypotheses 2010;74:859-861.

40 Jarboe EA, Folkins AK, Drapkin R, et al. Tubal and ovarian pathways to pelvic epithelial cancer: a pathological perspective. Histopathology 2008;53: 127-138.

41 Young RH, Scully RE. Invasive adenocarcinoma and related tumors of the uterine cervix. Semin Diagn Pathol 1990;7:205-227.

42 Gilks CB, Clement PB. Papillary serous adenocarcinoma of the uterine cervix: a report of three cases. Mod Pathol 1992;5:426-431.

43 Zhou C, Gilks CB, Hayes M, et al. Papillary serous carcinoma of the uterine cervix - a clinicopathologic study of 17 cases. Am J Surg Pathol 1998;22:113-120.

44 Harris CC 1995) Deichmann lecture-p53 tumor suppressor gene: at the crossroads of molecular carcinogenesis, molecular epidemiology and cancer risk assessment. Toxicol Lett 1995;82-3:1-7.

45 Kurman RJ, Shih IeM. Molecular pathogenesis and extraovarian origin of epithelial ovarian cancershifting the paradigm. Hum Pathol 2011;42:918-931.

46 Cheng L, Jones TD, McCarthy RP, et al. Molecular genetic evidence for a common clonal origin of urinary bladder small cell carcinoma and coexisting urothelial carcinoma. Am J Pathol 2005;166:1533-1539. 
47 Horn LC, Kafkova S, Leonhardt K, et al. Serous tubal in situ carcinoma (STIC) in primary peritoneal serous carcinomas. Int J Gynecol Pathol 2013;32:339-344.

48 Horn LC, Leonhardt K, Kafkova S, et al. Serous tubal in situ carcinoma (STIC) in tubal and primary peritoneal carcinomas. Virchows Arch 2012;461: S308-S309.

49 Folkins AK, Jarboe EA, Roh $\mathrm{MH}$, et al. Precursors to pelvic serous carcinoma and their clinical implications. Gynecol Oncol 2009;113:391-396. 